\title{
Relieving Pain with Acupuncture after Inguinal Surgeries
}

Department of Student Research Committee, *Department of Physiology, Jahrom University of Medical Sciences, Jahrom, Iran

\author{
Mohamed Amin Ghobadifar, MD, Safar Zarei, MS*, and Amir Hossein Heidarnezhad, MD
}

\section{LETTER TO EDITORS}

With great interest, we read a recently published article in the Korean Journal of Pain by Rahim Taghavi et al., entitled "The effect of acupuncture on relieving pain after inguinal surgeries" [1]. The authors reported an interesting investigation about the efficacy of acupuncture in reducing postoperative pain. They concluded that "After inguinal surgery, acupuncture in patients can reduce the need of analgesics, which also reduces the complications directly that may occur when analgesics are used in relieving pain postoperatively." Although this issue is an interesting topic in pain and traditional medicine, there are some concerns over the mentioned study that undermine the reported data making it difficult to form a definite conclusion.

The amount of cases was not estimated and the author should estimate the "power" of this study to compensate for any refusal of the data. Second, randomization in an experimental study is so important and critical. The method used for randomization (e.g. computer-based randomization table, etc.) was not reported in this study by the authors. The authors show the equal distribution of the demographic data between the two study groups in their study but they do not show any table that presents the values of the variables and their $P$ values to confirm their claim. This is also true for another part of their results in which they report that the dosage of the acupuncture group is significantly lower than that of the control group, or on the other hand, they show that the number of the subjects with adverse effects in the acupuncture group is significantly lower than that of the control group but they do not provide any $P$ values to confirm the reported data. Third, a participation fellow chart is necessary for clinical studies, according to CONSORT (Consolidated Standards of Reporting Trials) statement [2,3]. The authors show that they enrolled a total of 90 patients into their study and excluded those patients who had any history of opium addiction, inguinal surgery and interfering neurological diseases. However, in the analysis of the data, we see ninety patients who were enrolled at the onset of the study and no one was excluded which makes it necessary for the authors to show a participation fellow chart. Moreover, in the methods, the statistical analysis is poorly described and the authors do not report any $P$ values less than what were considered to be statistically significant.

Despite the variability in severity of pain such as off-and-on or steady, sharp or dull, localized, or all over the body [4], the stated kinds of pain reduced by the acupuncture are limited in this study. On the other hand, the drugs used for general anesthesia (fentanyl, atracurium and others) may have side effects such as nausea, vomit-

Received March 28, 2013. Accepted April 18, 2013.

Correspondence to: Mohamed Amin Ghobadifar, MD

Department of Student Research Committee, Medicine School, Jahrom University of Medical Sciences, Motahari Avenue, Jahrom, Iran Tel: +98-936-620-8078, Fax: +98-711-636-13-86, E-mail: amin_m505@yahoo.com

(c) This is an open-access article distributed under the terms of the Creative Commons Attribution Non-Commercial License (http:// creativecommons.org/licenses/by-nc/3.0/), which permits unrestricted non-commercial use, distribution, and reproduction in any medium, provided the original work is properly cited.

Copyright (c) The Korean Pain Society, 2013 
ing, and dizziness $[5,6]$ that make the conclusion about fewer side effects from acupuncture doubtful. We suggest that a study with accurate and powerful methods according to the CONSORT statement is necessary to fully investigate this topic. Further investigations in the methods and role of acupuncture in patients with the chief complaint of postoperative pain are needed.

\section{REFERENCES}

1. Taghavi R, Tabasi KT, Mogharabian N, Asadpour A, Golchian A. Mohamadi S, et al. The effect of acupuncture on relieving pain after inguinal surgeries. Korean J Pain 2013; 26: 46-50.

2. Schulz KF, Altman DG, Moher D; CONSORT Group. CONSORT 2010 statement: updated guidelines for reporting parallel group randomised trials. J Clin Epidemiol 2010; 63: 834-40.

3. Begg C, Cho M, Eastwood S, Horton R, Moher D, Olkin I, et al. Improving the quality of reporting of randomized controlled trials. The CONSORT statement. JAMA 1996; 276: 637-9.

4. Cho SK, Heiby EM, McCracken LM, Moon DE, Lee JH. Daily functioning in chronic pain: study of structural relations with posttraumatic stress disorder symptoms, pain intensity, and pain avoidance. Korean J Pain 2011; 24: 13-21.

5. Minkowitz HS, Yarmush J, Donnell MT, Tonner PH, Damaraju CV, Skowronski RJ. Safety and tolerability of fentanyl iontophoretic transdermal system: findings from a pooled data analysis of four clinical trials. J Opioid Manag 2010; 6: 203-10.

6. Warren-Stomberg M, Brattwall M, Jakobsson JG. Non-opioid analgesics for pain management following ambulatory surgery, a review. Minerva Anestesiol 2013 [in press]. 